\title{
Synthesis of $N$-benzoyl Amino Esters and $N$-benzoyl Amino Acids and their Antifungal Activity
}

Yureli Chiguils-Pérez ${ }^{1}$, Alejandro Israel Rodríguez-Hurtado ${ }^{2}$, Lemuel Pérez-Picaso ${ }^{1 *}$, Roxana Martínez-Pascual $^{1 *}$, María de los Ángeles Martínez-Rivera ${ }^{2}$, Emanuel Hernández-Núñez ${ }^{4}$ Omar Viñas-Bravo $^{1}$, Sharon Rosete-Luna ${ }^{3}$, Nelda Xanath Martínez-Galero ${ }^{1}$

${ }^{1}$ Centro de Investigaciones Científicas, Instituto de Química Aplicada, Universidad del Papaloapan, Tuxtepec 68301, Oaxaca, México.

${ }^{2}$ Laboratorio de Micología Médica, Escuela Nacional de Ciencias Biológicas, Instituto Politécnico Nacional, Ciudad de México, 11340, México.

${ }^{3}$ Facultad de Ciencias Químicas, Universidad Veracruzana, Oriente 6, Col. Rafael Alvarado, 94340, Orizaba, Veracruz.

${ }^{4}$ Departamento de Recursos del Mar, CINVESTAV-IPN Unidad Mérida, Mérida, 97310, Yucatán, México.

*Corresponding author: Roxana Martínez-Pascual, email: rpascual@unpa.edu.mx; Lemuel Pérez-Picaso, email: lemuelp@unpa.edu.mx; Tel.: +52 2878759240 ext 230.

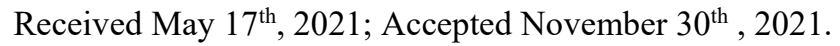

DOI: http://dx.doi.org/10.29356/jmcs.v66i1.1584

\begin{abstract}
A series of $N$-benzoyl amino esters and $N$-benzoyl amino acids were synthesized from commercially available amino acids (Val, Ile, Leu, Ala, Phe, Trp) and were evaluated for their antifungal activity against two filamentous fungi, A. fumigatus and $F$. temperatum. According to the in vitro assays, five compounds (5-7, 10, 13) exhibited relevant antifungal activity against $F$. temperatum and two compounds (5 and 7) showed remarkable activity against both fungi strains. Some structure-activity relationships were established regarding the side chain at $\mathrm{C} \alpha$ and the type of substituents on the aromatic ring in the benzoyl moiety. Docking calculations were performed in order to predict binding affinities between compounds prepared herein and fungal chitinase, a potential target against fungi; interactions involving the aromatic rings, the influence on the number of methyl substituents, and configurations on the $\alpha$-carbon have been analyzed.

Keywords: $N$-benzoyl amino esters; $N$-benzoyl amino acids; antifungal activity; A. fumigatus; $F$. temperatum.

Resumen. Una serie de derivados $N$-benzoilamino ésteres y $N$-benzoilaminoácidos, sintetizados a partir de aminoácidos disponibles comercialmente (Val, Ile, Leu, Ala, Phe, Trp), se evaluaron como agentes antifúngicos frente a dos hongos filamentosos, A. fumigatus y $F$. temperatum. De acuerdo con los ensayos in vitro, cinco compuestos $(5-7,10,13)$ exhibieron una actividad relevante contra $F$. temperatum y dos derivados $(5$ y 7$)$ mostraron una actividad notable contra ambas cepas. Algunas relaciones de estructura actividad permitieron observar el efecto de la cadena lateral del aminoácido, y de los sustituyentes del grupo benzoílo, en la actividad biológica. Se realizaron cálculos de acoplamiento molecular con el propósito de predecir afinidades de enlace entre los compuestos sintetizados y la enzima quitinasa, considerada un blanco molecular potencial. Se analizaron las interacciones que involucran anillos aromáticos, la influencia de los sustituyentes metilo, así como la configuración del $\mathrm{C} \alpha$.
\end{abstract}


Palabras clave: $N$-benzoilamino ésteres; $N$-benzoilaminoácidos; actividad antifúngica; A. fumigatus; $F$. temperatum.

\section{Introduction}

Human infections caused by fungi are common diseases in the population all over the world, the most frequent and relatively less serious can be fungal nail, skin or hair infections. Nevertheless, other diseases such as, aspergillosis, invasive candidiasis, mucormycosis, pneumocystis pneumonia, histoplasmosis, talaromycosis or C. neoformans infection, become mortal in immunocompromised patients whose population is still growing [1]. Fungal diseases also affect people who live or travel to certain areas, for example blastomycosis, histoplasmosis or the valley fever [2]. Recent studies show that, regarding fungal infections, over 150 million severe cases occur worldwide, causing 1.7 million deaths [3]. One major challenge about these infections is to accomplish an early diagnose so that an appropriate treatment can be started immediately. Another problem is the increasing resistance to several antimycotic drugs, such as fluconazole, amphotericin B, voriconazole [4,5] and the emerging caspofungin resistance [6]. Combination of these circumstances has made fungal infections to be considered by experts as a public health issue that must not be underestimated [7]. In comparison with antibacterial or antivirus drugs, fewer contributions have been made to the development of novel antifungal drugs. The clinical approval of new antifungal drugs has been more slowly; only isavuconazole has been marketed over the last decade [8]. In this context, it becomes necessary to search for new antifungal agents with different modes of action, enhanced activity and low toxicity in order to have more therapeutic options.

It has been known for more than a century that sodium benzoate exhibits both antifungal and antimicrobial activities, and because of these properties it is still used as food or pharmaceuticals preservative [9]. Furthermore, other weak acids as, propionic, sorbic, cinnamic, and salicylic and also some esters have shown antimycotic activities [10-12].

Besides, amino acids play an important role in the metabolism of living organisms and are important building blocks to construct peptides and proteins. Amino acid residues are incorporated into numerous bioactive compounds in order to improve biocompatibility and to decrease toxicity [13-15]. They are also used as starting materials for the synthesis of heterocycles [16] and other bioactive compounds [17,18]. Several amino acid derivatives have been reported for their biological activities, such as $\mathrm{N}$-acylamino acids [19], $\mathrm{N}$ acylamino esters [20], $\mathrm{N}$-tryptophan esters [21], $\mathrm{N}$-amino acid esters of salicylanilides [22], $\mathrm{N}$-acetamido benzimidazole derivatives [23], or amino acids linked to triazoles [24]. In the present study, we report the synthesis of a series of $N$-benzoyl amino acids and $N$-benzoylamino esters and their biological evaluation as antifungal agents against two filamentous fungi, Fusarium temperatum and Aspergillus fumigatus. The set of analogs were synthesized by a coupling reaction between a benzoic acid derivative and methyl ester amino acid or by $\mathrm{N}$-acylation of an amino acid with benzoic anhydride.

\section{Experimental}

\section{Materials and Methods}

Reagents were used as received from Sigma-Aldrich without further purification. NMR spectra were recorded on a Varian Mercury spectrometer $\left(400 \mathrm{MHz}\right.$ for ${ }^{1} \mathrm{H}, 100.6 \mathrm{MHz}$ for $\left.{ }^{13} \mathrm{C}\right)$. Chemical shifts are quoted in ppm $(\delta)$ and spectra were referenced to the residual solvent signals ( 7.27 and $77.2 \mathrm{ppm}$ for $\mathrm{CDCl}_{3}$ ). Coupling constants $(J)$ are expressed in Hertz $(\mathrm{Hz})$. Multiplicities are recorded as follows: $\mathrm{s}=$ singlet, $\mathrm{d}=\mathrm{doublet}, \mathrm{t}=$ triplet, $\mathrm{dd}=$ doublet of doublets, $\mathrm{td}=$ triplet of doublets, $\mathrm{dh}=$ doublet of heptuplets, brs= broad singlet, $\mathrm{q}=$ quartet, and $\mathrm{m}=$ multiplet. High-resolution mass spectra (HRMS) were recorded on a JEOL JMStation-JM 700 mass spectrometer at $70 \mathrm{eV}$ in a matrix of glycerol or in a Synapt G2-Si (Waters) spectrometer equipped with electrospray ion source (ESI), single quadrupole mass filter and time of flight mass analyzer (Q-TOF). IR 
spectra were acquired on FTIR Perkin Elmer Spectrum 100 (range: 4000-600 $\mathrm{cm}^{-1}$ ). Melting points were measured in a Mel-Temp apparatus and were not corrected. Analytical TLC was performed using pre-coated silica gel plates $60 \mathrm{~F}_{254}$, and chromatographic columns were carried out on Davisil ${ }^{\mathrm{TM}}$ grade 633 silica gel (200425 mesh).

\section{General Procedure for the synthesis of $\alpha$-aminoesters}

Trimethylsilane chloride ( $2 \mathrm{mmol}$ ) was added to a solution of the corresponding amino acid (1 mmol) in $\mathrm{MeOH}(5 \mathrm{~mL})$. The solution was stirred for $12 \mathrm{~h}$ at room temperature. Then, the solvent was evaporated under reduced pressure. The residue was characterized and used without further purification in the following reaction.

\section{General Procedure for the synthesis of $N$-benzoylamino acid methyl esters (1-7, 9-17 and 19)}

A solution of the corresponding $\alpha$-aminoester $(1 \mathrm{mmol})$, the carboxylic acid $(1 \mathrm{mmol})$, DMAP $(0.1$ mmol), $N$-(3-Dimethylaminopropyl)- $N$ '-ethylcarbodiimide hydrochloride, $(1.5 \mathrm{mmol})$ and triethylamine $(2$ mmol) in $\mathrm{CH}_{2} \mathrm{Cl}_{2}(10 \mathrm{~mL})$ was stirred at room temperature overnight. Then, $\mathrm{CH}_{2} \mathrm{Cl}_{2}(20 \mathrm{~mL})$ and $\mathrm{NH}_{4} \mathrm{Cl}$ sat. aqueous solution $(10 \mathrm{~mL})$ was added to the mixture. The organic layer was separated, and the aqueous phase was extracted with $\mathrm{CH}_{2} \mathrm{Cl}_{2}(3 \times 20 \mathrm{~mL})$. The combined organic fractions were dried over $\mathrm{Na}_{2} \mathrm{SO}_{4}$, filtrated, and the filtrate was evaporated under reduced pressure; the residue was purified as indicated below.

$N$-Benzoyl-L-valine methyl ester (1). Purification by column chromatography (hexane-EtOAc, 80:20) gave $\mathbf{1}$ as a white solid (200 mg, 85\% yield); mp 110.9-111.4 ${ }^{\circ} \mathrm{C}$. Characterization of $\mathbf{1}$ has been previously reported in the literature [25a]

$\mathrm{N}$-(3-methoxybenzoyl)-L-valine methyl ester (2). Purification by column chromatography (hexaneEtOAc, 80:20) gave 2 as a white solid (199 mg, $75 \%$ yield); mp 80.9-81.5 ${ }^{\circ} \mathrm{C}$; IR $\left(\mathrm{cm}^{-1}\right): 3224(\mathrm{NH}), 1719$ $\left(\mathrm{CO}_{2} \mathrm{R}\right), 1620(\mathrm{CONH}), 1522$; $\mathrm{NMR}{ }^{1} \mathrm{H}\left(400 \mathrm{MHz}, \mathrm{CDCl}_{3}\right) \delta: 7.40-7.27\left(3 \mathrm{H}, \mathrm{m}, \mathrm{H}_{\mathrm{Ar}}\right), 7.01(1 \mathrm{H}, \mathrm{ddd}, J=8.0$, 2.6, $\left.1.1 \mathrm{~Hz}, \mathrm{H}_{\mathrm{Ar}}\right), 6.95(1 \mathrm{H}, \mathrm{d}, J=8.7 \mathrm{~Hz}, \mathrm{NH}), 4.74\left(1 \mathrm{H}, \mathrm{dd}, J=8.7,5.4 \mathrm{~Hz}, \mathrm{H}_{\alpha}\right), 3.80\left(3 \mathrm{H}, \mathrm{s}, \mathrm{CH}_{3} \mathrm{OAr}\right), 3.75$ $\left(3 \mathrm{H}, \mathrm{s}, \mathrm{CH}_{3} \mathrm{O}\right), 2.27\left(1 \mathrm{H}, \mathrm{dh}, J=6.8,5.4 \mathrm{~Hz}, \mathrm{H}_{\beta}\right), 1.00\left(3 \mathrm{H}, \mathrm{d}, J=6.8 \mathrm{~Hz}, \mathrm{H}_{\gamma}\right), 0.98\left(3 \mathrm{H}, \mathrm{d}, J=6.8 \mathrm{~Hz}, \mathrm{H}_{\gamma}\right)$; NMR ${ }^{13} \mathrm{C}\left(101 \mathrm{MHz}, \mathrm{CDCl}_{3}\right) \delta: 172.5(\mathrm{COOR}), 167.2(\mathrm{CON}), 199.5\left(\mathrm{C}_{\mathrm{Ar}}\right), 136.4\left(\mathrm{C}_{\mathrm{Ar}}\right), 129.4\left(\mathrm{C}_{\mathrm{Ar}}\right), 118.8\left(\mathrm{C}_{\mathrm{Ar}}\right), 117.7$ $\left.\left(\mathrm{C}_{\mathrm{Ar}}\right), 112.5\left(\mathrm{C}_{\mathrm{Ar}}\right), 57.6\left(\mathrm{CH}_{3} \mathrm{OAr}\right), 55.3\left(\mathrm{CH}_{3} \mathrm{O}\right), 52.1\left(\mathrm{C}_{\alpha}\right), 31.3\left(\mathrm{C}_{\beta}\right), 18.9\left(\mathrm{C}_{\gamma}\right), 18.0\left(\mathrm{C}_{\gamma}\right)^{\prime}\right)$. ESI-HRMS calcd. for $\mathrm{C}_{14} \mathrm{H}_{20} \mathrm{NO}_{4}[\mathrm{M}+\mathrm{H}]^{+}: 266.1392$, found: 266.1378 .

$\mathrm{N}$-(3,4-dimethoxybenzoyl)-L-valine methyl ester (3). Purification by column chromatography (hexaneEtOAc, 60:40) gave 3 as a white solid $\left(221 \mathrm{~g}, 75 \%\right.$ yield); mp 90.5-91.0 ${ }^{\circ} \mathrm{C}$; IR $\left(\mathrm{cm}^{-1}\right): 3270(\mathrm{NH}), 1732$ $\left(\mathrm{CO}_{2} \mathrm{R}\right), 1620(\mathrm{CONH}) ; \mathrm{NMR}^{1} \mathrm{H}\left(400 \mathrm{MHz}, \mathrm{CDCl}_{3}\right) \delta: 7.44\left(1 \mathrm{H}, \mathrm{d}, J=2.0 \mathrm{~Hz}, \mathrm{H}_{\mathrm{Ar}}\right), 7.34(1 \mathrm{H}, \mathrm{dd}, J=8.4,2.0$ $\left.\mathrm{Hz}, \mathrm{H}_{\mathrm{Ar}}\right), 6.88\left(1 \mathrm{H}, \mathrm{d}, J=8.4 \mathrm{~Hz}, \mathrm{H}_{\mathrm{Ar}}\right), 6.57(1 \mathrm{H}, \mathrm{d}, J=8.6 \mathrm{~Hz}, \mathrm{NH}), 4.77\left(1 \mathrm{H}, \mathrm{dd}, J=8.6,5.0 \mathrm{~Hz}, \mathrm{H}_{\alpha}\right), 3.94(3 \mathrm{H}$, $\left.\mathrm{s}, 3-\mathrm{CH}_{3} \mathrm{O}\right), 3.93\left(3 \mathrm{H}, \mathrm{s}, 4-\mathrm{CH}_{3} \mathrm{O}\right), 3.78\left(3 \mathrm{H}, \mathrm{s}, \mathrm{CH}_{3} \mathrm{O}\right), 2.28\left(1 \mathrm{H}, \mathrm{dh}, J=6.9,5.0 \mathrm{~Hz}, \mathrm{H}_{\beta}\right), 1.02(3 \mathrm{H}, \mathrm{d}, J=6.9$ $\left.\mathrm{Hz}, \mathrm{H}_{\gamma}\right), 0.99\left(3 \mathrm{H}, \mathrm{d}, J=6.9 \mathrm{~Hz}, \mathrm{H}_{\gamma}\right)$; $\mathrm{NMR}^{13} \mathrm{C}\left(101 \mathrm{MHz}, \mathrm{CDCl}_{3}\right) \delta: 173.0(\mathrm{COOR}), 167.0(\mathrm{CON}), 152.2\left(\mathrm{C}_{\mathrm{Ar}}\right)$, $149.3\left(\mathrm{C}_{\mathrm{Ar}}\right), 127.0\left(\mathrm{C}_{\mathrm{Ar}}\right), 119.6\left(\mathrm{C}_{\mathrm{Ar}}\right), 111.0\left(\mathrm{C}_{\mathrm{Ar}}\right), 110.5\left(\mathrm{C}_{\mathrm{Ar}}\right), 57.6\left(3-\mathrm{CH}_{3} \mathrm{O}\right), 56.3\left(4-\mathrm{CH}_{3} \mathrm{O}\right), 56.2\left(\mathrm{CH}_{3} \mathrm{O}\right)$, $52.4\left(\mathrm{C}_{\alpha}\right), 31.9\left(\mathrm{C}_{\beta}\right), 19.2\left(\mathrm{C}_{\gamma}\right), 18.2\left(\mathrm{C}_{\gamma^{\prime}}\right)$. ESI-HRMS calcd. for $\mathrm{C}_{15} \mathrm{H}_{22} \mathrm{NO}_{5}[\mathrm{M}+\mathrm{H}]^{+}: 296.1498$, found: 296.1501 .

$\mathrm{N}$-[2-(4-hydroxyphenyl)acetyl]-L-valine methyl ester (4). Purification by column chromatography (hexane-EtOAc, 50:50) gave 4 as a yellow solid $\left(216 \mathrm{mg}, 86 \%\right.$ yield), $\mathrm{mp} 111.1-112.7^{\circ} \mathrm{C}$. IR $\left(\mathrm{cm}^{-1}\right): 3290$ $(\mathrm{OH}), 1752\left(\mathrm{CO}_{2} \mathrm{R}\right), 1600(\mathrm{CONH})$. NMR ${ }^{1} \mathrm{H}\left(400 \mathrm{MHz}, \mathrm{CDCl}_{3}\right) \delta: 8.14(1 \mathrm{H}$, brs, $\mathrm{OH}), 7.05(2 \mathrm{H}, \mathrm{d}, J=8.5 \mathrm{~Hz}$, $\left.\mathrm{H}_{\mathrm{Ar}}\right), 6.78\left(2 \mathrm{H}, \mathrm{dd}, J=8.5,2.4 \mathrm{~Hz}, \mathrm{H}_{\mathrm{Ar}}\right), 6.29(1 \mathrm{H}, \mathrm{d}, J=8.8 \mathrm{~Hz}, \mathrm{NH}), 4.51\left(1 \mathrm{H}, \mathrm{dd}, J=8.8,5.2 \mathrm{~Hz}, \mathrm{H}_{\alpha}\right), 3.67$ $\left(3 \mathrm{H}, \mathrm{s}, \mathrm{CH}_{3} \mathrm{O}\right), 3.52\left(2 \mathrm{H}, \mathrm{s}, \mathrm{CH}_{2} \mathrm{Ph}\right), 2.07\left(1 \mathrm{H}, \mathrm{dh}, J=6.8,5.2 \mathrm{~Hz}, \mathrm{H}_{\beta}\right), 0.84\left(3 \mathrm{H}, \mathrm{d}, J=6.8 \mathrm{~Hz}, \mathrm{H}_{\gamma}\right), 0.76(3 \mathrm{H}, \mathrm{d}$, $\left.J=6.8 \mathrm{~Hz}, \mathrm{H}_{\gamma^{\prime}}\right) . \mathrm{NMR}^{13} \mathrm{C}\left(101 \mathrm{MHz}, \mathrm{CDCl}_{3}\right) \delta: 172.8(\mathrm{COOR}), 172.5(\mathrm{CON}), 156.2\left(\mathrm{C}_{\mathrm{Ar}}\right), 130.5\left(\mathrm{C}_{\mathrm{Ar}}\right), 125.2$ $\left(\mathrm{C}_{\mathrm{Ar}}\right), 116.1\left(\mathrm{C}_{\mathrm{Ar}}\right), 57.3\left(\mathrm{CH}_{3} \mathrm{O}\right), 52.4\left(\mathrm{C}_{\alpha}\right), 42.6\left(\mathrm{CH}_{2} \mathrm{Ph}\right), 31.1\left(\mathrm{C}_{\beta}\right), 19.0\left(\mathrm{C}_{\gamma}\right), 17.7\left(\mathrm{C}_{\gamma}\right)$. ESI-HRMS calcd. for $\mathrm{C}_{14} \mathrm{H}_{20} \mathrm{NO}_{4}[\mathrm{M}+\mathrm{H}]^{+}: 266.1392$ found: 266.1381 
$N$-Benzoyl-D-valine methyl ester (5). Purification by column chromatography (hexane-EtOAc, 80:20) gave 5 as a white solid (200 mg, $85 \%$ yield); $\mathrm{mp} 110.9-111.4{ }^{\circ} \mathrm{C}$; Characterization of $\mathbf{5}$ has been previously reported in the literature [25b].

$\mathrm{N}$-(4-methylbenzoyl)-L-valine methyl ester (6). Purification by column chromatography (hexaneEtOAc, 50:50) gave 6 as a white solid $\left(246 \mathrm{mg}, 99 \%\right.$ yield); $\mathrm{mp} 93-96^{\circ} \mathrm{C}$; IR $\left(\mathrm{cm}^{-1}\right): 3326(\mathrm{NH}), 1741\left(\mathrm{CO}_{2} \mathrm{R}\right)$, $1634(\mathrm{CONH}) . \mathrm{NMR}^{1} \mathrm{H}\left(\mathrm{CDCl}_{3}\right) \delta: 7.72\left(2 \mathrm{H}, \mathrm{dd}, J=8.4,0.6 \mathrm{~Hz}, \mathrm{H}_{\mathrm{Ar}}\right), 7.24\left(2 \mathrm{H}, \mathrm{dd}, J=8.4,0.6 \mathrm{~Hz}, \mathrm{H}_{\mathrm{Ar}}\right), 6.66$ $(1 \mathrm{H}, \mathrm{d}, J=8.7 \mathrm{~Hz}, \mathrm{NH}), 4.78\left(1 \mathrm{H}, \mathrm{dd}, J=8.7,5.0 \mathrm{~Hz}, \mathrm{H}_{\alpha}\right), 3.77\left(3 \mathrm{H}, \mathrm{s}, \mathrm{CH}_{3} \mathrm{O}\right), 2.40\left(3 \mathrm{H}, \mathrm{s}, \mathrm{CH}_{3}-\mathrm{Ar}\right), 2.27(1 \mathrm{H}$,

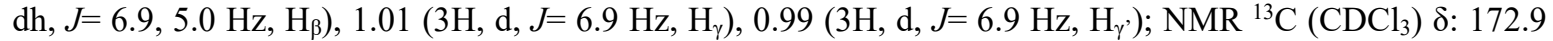
$(\mathrm{COOR}), 167.4(\mathrm{CON}), 142.3\left(\mathrm{C}_{\mathrm{Ar}}\right), 131.4\left(\mathrm{C}_{\mathrm{Ar}}\right), 129.4\left(\mathrm{C}_{\mathrm{Ar}}\right), 127.2\left(\mathrm{C}_{\mathrm{Ar}}\right), 57.5\left(\mathrm{C}_{\alpha}\right), 52.4\left(\mathrm{CH}_{3} \mathrm{O}\right), 31.8\left(\mathrm{C}_{\beta}\right)$, $21.6\left(\mathrm{CH}_{3}-\mathrm{Ar}\right), 19.1\left(\mathrm{C}_{\gamma}\right), 18.1\left(\mathrm{C}_{\gamma^{\prime}}\right)$. ESI-HRMS calcd. for $\mathrm{C}_{14} \mathrm{H}_{20} \mathrm{NO}_{3}[\mathrm{M}+\mathrm{H}]^{+}: 250.1443$, found: 250.1442 .

$\mathrm{N}$-(2,4,6-trimethylbenzoyl)-L-valine methyl ester (7). Purification by column chromatography (hexane-EtOAc, 60:40) gave 7 as a white solid $\left(100 \mathrm{mg}, 36 \%\right.$ yield); $\mathrm{mp} 78-79^{\circ} \mathrm{C}$; IR $\left(\mathrm{cm}^{-1}\right): 1709\left(\mathrm{CO}_{2} \mathrm{R}\right)$, $1660(\mathrm{CONH})$; $\mathrm{NMR}{ }^{1} \mathrm{H}\left(\mathrm{CDCl}_{3}\right) \delta: 6.86\left(2 \mathrm{H}, \mathrm{s}, \mathrm{H}_{\mathrm{Ar}}\right), 6.11(1 \mathrm{H}, \mathrm{d}, J=8.8 \mathrm{~Hz}, \mathrm{NH}), 4.80(1 \mathrm{H}, \mathrm{dd}, J=8.8,4.6$ $\left.\mathrm{Hz}, \mathrm{H}_{\alpha}\right), 3.77\left(3 \mathrm{H}, \mathrm{s}, \mathrm{CH}_{3} \mathrm{O}\right), 2.36-2.23\left(1 \mathrm{H}, \mathrm{m}, \mathrm{H}_{\beta}\right), 2.31\left(6 \mathrm{H}, \mathrm{s}, o-\mathrm{CH}_{3}-\mathrm{Ar}\right) 2.28\left(3 \mathrm{H}, \mathrm{s}, p-\mathrm{CH}_{3}-\mathrm{Ar}\right), 1.05(3 \mathrm{H}$, $\left.\mathrm{d}, J=6.9 \mathrm{~Hz}, \mathrm{H}_{\gamma}\right), 0.93\left(3 \mathrm{H}, \mathrm{d}, J=6.9 \mathrm{~Hz}, \mathrm{H}_{\gamma}\right) ; \mathrm{NMR}^{13} \mathrm{C}\left(\mathrm{CDCl}_{3}\right) \delta: 172.5(\mathrm{COOR}), 170.7(\mathrm{CON}), 138.8\left(\mathrm{C}_{\mathrm{Ar}}\right)$, $134.7\left(\mathrm{C}_{\mathrm{Ar}}\right), 134.5\left(\mathrm{C}_{\mathrm{Ar}}\right), 128.4\left(\mathrm{C}_{\mathrm{Ar}}\right), 57.1\left(\mathrm{C}_{\alpha}\right), 52.4\left(\mathrm{CH}_{3} \mathrm{O}\right), 31.2\left(\mathrm{C}_{\beta}\right), 21.3\left(p-\mathrm{CH}_{3}-\mathrm{Ar}\right), 19.4\left(\mathrm{C}_{\gamma}\right), 19.4(o-$ $\left.\mathrm{CH}_{3}-\mathrm{Ar}\right), 17.9\left(\mathrm{C}_{\gamma^{\prime}}\right)$. ESI-HRMS calcd. for $\mathrm{C}_{16} \mathrm{H}_{23} \mathrm{NO}_{3} \mathrm{Na}[\mathrm{M}+\mathrm{Na}]^{+}: 300.1576$, found: 300.1574 .

N-Benzoyl-L-tryptophan methyl ester (9). Purification by column chromatography (hexane-EtOAc, $70: 30$ ) gave 9 as a yellow solid ( $145 \mathrm{mg}, 45 \%$ yield); $\mathrm{mp} 107-109^{\circ} \mathrm{C}$. Characterization of 9 has been previously reported in the literature $[25 \mathrm{c}]$.

$\mathrm{N}$-(2-hydroxybenzoyl)-L-tryptophan methyl ester (10). Purification by column chromatography (hexane-EtOAc, 80:20) gave 10 as a yellow oil (196 mg, $58 \%$ yield); IR $\left(\mathrm{cm}^{-1}\right): 3406(\mathrm{NH}), 1731\left(\mathrm{CO}_{2} \mathrm{R}\right)$, $1638(\mathrm{CONH})$; NMR ${ }^{1} \mathrm{H}\left(\mathrm{CDCl}_{3}\right) \delta: 12.15(1 \mathrm{H}, \mathrm{s}, \mathrm{OH}), 8.18\left(1 \mathrm{H}\right.$, brs, $\left.\mathrm{NH}_{\text {indol }}\right), 7.53(1 \mathrm{H}, \mathrm{dd}, J=8.0,0.8 \mathrm{~Hz}$, $\left.\mathrm{H}_{\mathrm{Ar}}\right), 7.39-7.35\left(2 \mathrm{H}, \mathrm{m}, \mathrm{H}_{\mathrm{Ar}}\right), 7.24-7.07\left(3 \mathrm{H}, \mathrm{m}, \mathrm{H}_{\mathrm{Ar}}\right), 7.00\left(1 \mathrm{H}, \mathrm{d}, J=2.4 \mathrm{~Hz}, \mathrm{H}_{\mathrm{Ar}}\right), 6.97(1 \mathrm{H}, \mathrm{dd}, J=8.4,1.2$ $\left.\mathrm{Hz}, \mathrm{H}_{\mathrm{Ar}}\right), 6.85(1 \mathrm{H}, \mathrm{d}, J=7.4 \mathrm{~Hz}, \mathrm{NH}), 6.75\left(1 \mathrm{H}, \mathrm{ddd}, J=8.2,7.3,1.2 \mathrm{~Hz}, \mathrm{H}_{\mathrm{Ar}}\right), 5.10(1 \mathrm{H}, \mathrm{dt}, J=7.4,5.2 \mathrm{~Hz}$, $\left.\mathrm{H}_{\alpha}\right), 3.74\left(3 \mathrm{H}, \mathrm{s}, \mathrm{CH}_{3} \mathrm{O}\right), 3.47\left(1 \mathrm{H}, \mathrm{dd}, J=20.0,5.2 \mathrm{~Hz}, \mathrm{H}_{\beta}\right), 3.42\left(1 \mathrm{H}, \mathrm{dd}, J=20.0,5.2 \mathrm{~Hz}, \mathrm{H}_{\beta}\right)$; NMR ${ }^{13} \mathrm{C}$ $\left(\mathrm{CDCl}_{3}\right) \delta: 172.2(\mathrm{COOR}), 169.7(\mathrm{CON}), 161.6\left(\mathrm{C}_{\mathrm{Ar}-\mathrm{OH}}\right), 136.3\left(\mathrm{C}_{\mathrm{Ar}}\right), 134.6\left(\mathrm{C}_{\mathrm{Ar}}\right), 127.6\left(\mathrm{C}_{\mathrm{Ar}}\right), 126.0\left(\mathrm{C}_{\mathrm{Ar}}\right)$, $123.1\left(\mathrm{C}_{\mathrm{Ar}}\right), 122.5\left(\mathrm{C}_{\mathrm{Ar}}\right), 119.9\left(\mathrm{C}_{\mathrm{Ar}}\right), 118.9\left(\mathrm{C}_{\mathrm{Ar}}\right), 118.5\left(\mathrm{C}_{\mathrm{Ar}}\right), 113.9\left(\mathrm{C}_{\mathrm{Ar}}\right), 111.6\left(\mathrm{C}_{\mathrm{Ar}}\right), 109.6\left(\mathrm{C}_{\mathrm{Ar}}\right), 53.2\left(\mathrm{C}_{\alpha}\right)$, $52.7\left(\mathrm{CH}_{3} \mathrm{O}\right), 27.6\left(\mathrm{C}_{\beta}\right)$. HRMS-FAB calcd. for $\mathrm{C}_{19} \mathrm{H}_{19} \mathrm{~N}_{2} \mathrm{O}_{4}[\mathrm{M}+\mathrm{H}]^{+}$: 339.1345, found: 339.1351.

N-(2-hydroxynicotinoyl)-L-tryptophan methyl ester (11). Purification by column chromatography (EtOAc, $100 \%$ ) gave 11 as white solid (146 g, $43 \%$ yield); mp $113-117{ }^{\circ} \mathrm{C}$; IR $\left(\mathrm{cm}^{-1}\right): 3242(\mathrm{NH}), 1737$ $\left(\mathrm{CO}_{2} \mathrm{R}\right), 1661(\mathrm{CONH}) ; \mathrm{NMR}^{1} \mathrm{H}\left(\mathrm{CDCl}_{3}\right) \delta: 11.97(1 \mathrm{H}, \mathrm{brs}, \mathrm{OH}), 10.13(1 \mathrm{H}, \mathrm{d}, J=7.2 \mathrm{~Hz}, \mathrm{NH}), 8.47(1 \mathrm{H}, \mathrm{dd}$, $\left.J=7.2,2.0 \mathrm{~Hz}, \mathrm{H}_{\mathrm{Ar}}\right), 8.38(1 \mathrm{H}$, brs, $\mathrm{NH}), 7.56\left(1 \mathrm{H}, \mathrm{d}, J=8.2 \mathrm{~Hz}, \mathrm{H}_{\mathrm{Ar}}\right), 7.26-7.23\left(1 \mathrm{H}, \mathrm{m}, \mathrm{H}_{\mathrm{Ar}}\right), 7.14-7.03(4 \mathrm{H}$, $\left.\mathrm{m}, \mathrm{H}_{\mathrm{Ar}}\right), 6.30\left(1 \mathrm{H}, \mathrm{dd}, J=7.2,6.4 \mathrm{~Hz}, \mathrm{H}_{\mathrm{Ar}}\right), 5.11-5.05\left(1 \mathrm{H}, \mathrm{m}, \mathrm{H}_{\alpha}\right), 3.70\left(3 \mathrm{H}, \mathrm{s}, \mathrm{CH}_{3} \mathrm{O}\right), 3.42(1 \mathrm{H}, \mathrm{dd}, J=14.8$, $\left.6.2 \mathrm{~Hz}, \mathrm{H}_{\beta}\right), 3.38\left(1 \mathrm{H}, \mathrm{dd}, J=14.8,5.3 \mathrm{~Hz}, \mathrm{H}_{\beta}\right)$; $\mathrm{NMR}{ }^{13} \mathrm{C}\left(\mathrm{CDCl}_{3}\right) \delta: 172.9(\mathrm{COOR}), 163.8\left(\mathrm{C}_{\mathrm{Ar}-\mathrm{OH}}\right), 163.4$ $(\mathrm{CO}-\mathrm{NH}), 145.4\left(\mathrm{C}_{\mathrm{Ar}}\right), 138.4\left(\mathrm{C}_{\mathrm{Ar}}\right), 136.2\left(\mathrm{C}_{\mathrm{Ar}}\right), 127.5\left(\mathrm{C}_{\mathrm{Ar}}\right), 123.5\left(\mathrm{C}_{\mathrm{Ar}}\right), 122.2\left(\mathrm{C}_{\mathrm{Ar}}\right), 120.7\left(\mathrm{C}_{\mathrm{Ar}}\right), 119.6\left(\mathrm{C}_{\mathrm{Ar}}\right)$, $118.7\left(\mathrm{C}_{\mathrm{Ar}}\right), 111.5\left(\mathrm{C}_{\mathrm{Ar}}\right), 110.0\left(\mathrm{C}_{\mathrm{Ar}}\right), 107.8\left(\mathrm{C}_{\mathrm{Ar}}\right), 53.4\left(\mathrm{C}_{\alpha}\right), 52.6\left(\mathrm{CH}_{3} \mathrm{O}\right), 27.8\left(\mathrm{C}_{\beta}\right)$. HRMS-FAB calcd. for $\mathrm{C}_{18} \mathrm{H}_{18} \mathrm{~N}_{3} \mathrm{O}_{4}[\mathrm{M}+\mathrm{H}]^{+}: 340.1297$, found: 340.1315 .

$\mathrm{N}$-(2-chloronicotinoyl)-L-tryptophan methyl ester (12). Purification by column chromatography (hexane-EtOAc, 60:40) gave 12 as brown oil (100 mg, $28 \%$ yield); mp 113-117 ${ }^{\circ} \mathrm{C}$; IR $\left(\mathrm{cm}^{-1}\right): 3303(\mathrm{NH})$, $1738\left(\mathrm{CO}_{2} \mathrm{R}\right), 1650(\mathrm{CONH}) ; \mathrm{NMR}^{1} \mathrm{H}\left(\mathrm{CDCl}_{3}\right) \delta: 8.46\left(1 \mathrm{H}\right.$, brs, $\left.\mathrm{NH}_{\text {Indol }}\right), 8.37\left(1 \mathrm{H}, \mathrm{d}, J=2.2 \mathrm{~Hz}, \mathrm{H}_{\mathrm{Ar}}\right), 7.91$ $\left(1 \mathrm{H}, \mathrm{d}, J=7.5 \mathrm{~Hz}, \mathrm{H}_{\mathrm{Ar}}\right), 7.54\left(1 \mathrm{H}, \mathrm{d}, J=8.0 \mathrm{~Hz}, \mathrm{H}_{\mathrm{Ar}}\right), 7.31\left(1 \mathrm{H}, \mathrm{d}, J=8.0 \mathrm{~Hz}, \mathrm{H}_{\mathrm{Ar}}\right), 7.25-7.20\left(1 \mathrm{H}, \mathrm{m}, \mathrm{H}_{\mathrm{Ar}}\right)$, 7.18-7.13 $\left(1 \mathrm{H}, \mathrm{m}, \mathrm{H}_{\mathrm{Ar}}\right), 7.11-7.02\left(3 \mathrm{H}, \mathrm{m}, \mathrm{H}_{\mathrm{Ar}}\right), 5.11\left(1 \mathrm{H}, \mathrm{dt}, J=12.9,5.6 \mathrm{~Hz}, \mathrm{H}_{\alpha}\right), 3.73\left(3 \mathrm{H}, \mathrm{s}, \mathrm{CH}_{3} \mathrm{O}\right), 3.49$ $\left(1 \mathrm{H}, \mathrm{dd}, J=14.8,5.6 \mathrm{~Hz}, \mathrm{H}_{\beta}\right), 3.41\left(1 \mathrm{H}, \mathrm{dd}, J=14.8,5.6 \mathrm{~Hz}, \mathrm{H}_{\beta}\right) ; \mathrm{NMR}^{13} \mathrm{C}\left(\mathrm{CDCl}_{3}\right) \delta: 172.1$ (COOR), 164.5 
$(\mathrm{CON}), 151.1\left(\mathrm{C}_{\mathrm{Ar}}\right), 147.5\left(\mathrm{C}_{\mathrm{Ar}}\right), 139.8\left(\mathrm{C}_{\mathrm{Ar}}\right), 136.3\left(\mathrm{C}_{\mathrm{Ar}}\right), 130.8\left(\mathrm{C}_{\mathrm{Ar}}\right), 127.6\left(\mathrm{C}_{\mathrm{Ar}}\right), 123.2\left(\mathrm{C}_{\mathrm{Ar}}\right), 122.8\left(\mathrm{C}_{\mathrm{Ar}}\right)$, 122.4 $\left(\mathrm{C}_{\mathrm{Ar}}\right), 119.8\left(\mathrm{C}_{\mathrm{Ar}}\right), 118.6\left(\mathrm{C}_{\mathrm{Ar}}\right), 111.5\left(\mathrm{C}_{\mathrm{Ar}}\right), 109.6\left(\mathrm{C}_{\mathrm{Ar}}\right), 54.0\left(\mathrm{C}_{\alpha}\right), 52.8\left(\mathrm{CH}_{3} \mathrm{O}\right), 27.6\left(\mathrm{C}_{\beta}\right)$. HRMS-FAB calcd. for $\mathrm{C}_{18} \mathrm{H}_{17} \mathrm{ClN}_{3} \mathrm{O}_{3}[\mathrm{M}+\mathrm{H}]^{+}: 358.0958$, found: 358.0945 .

$\mathrm{N}$-(4-methylbenzoyl)-l-tryptophan methyl ester (13). Purification by column chromatography (hexaneEtOAc, 80:20) gave 13 as a yellow solid $\left(255 \mathrm{mg}, 76 \%\right.$ yield); mp $117-119{ }^{\circ} \mathrm{C}$; IR $\left(\mathrm{cm}^{-1}\right): 3360(\mathrm{NH}), 1731$ $\left(\mathrm{CO}_{2} \mathrm{R}\right), 1633(\mathrm{CONH}) ; \mathrm{NMR}^{1} \mathrm{H}\left(\mathrm{CDCl}_{3}\right) \delta: 8.81\left(1 \mathrm{H}, \mathrm{s}, \mathrm{NH}_{\text {Indol }}\right), 7.54\left(2 \mathrm{H}, \mathrm{d}, J=8.2 \mathrm{~Hz}, \mathrm{H}_{\mathrm{Ar}}\right), 7.50(1 \mathrm{H}, \mathrm{d}, J=$ $\left.7.7 \mathrm{~Hz}, \mathrm{H}_{\mathrm{Ar}}\right), 7.26\left(1 \mathrm{H}, \mathrm{dd}, J=8.9,0.7 \mathrm{~Hz}, \mathrm{H}_{\mathrm{Ar}}\right), 7.15-7.02\left(3 \mathrm{H}, \mathrm{m}, \mathrm{H}_{\mathrm{Ar}}\right) 6.90\left(1 \mathrm{H}, \mathrm{d}, J=2.4 \mathrm{~Hz}, \mathrm{H}_{\mathrm{Ar}}\right), 6.80(1 \mathrm{H}$, d, $J=7.6 \mathrm{~Hz}, \mathrm{NH}), 5.11\left(1 \mathrm{H}, \mathrm{dt}, J=7.6,5.2, \mathrm{~Hz}, \mathrm{H}_{\alpha}\right), 3.64\left(3 \mathrm{H}, \mathrm{s}, \mathrm{CH}_{3} \mathrm{O}\right), 3.41\left(1 \mathrm{H}, \mathrm{dd}, J=16.4,5.2 \mathrm{~Hz}, \mathrm{H}_{\beta}\right)$, $3.38\left(1 \mathrm{H}, \mathrm{dd}, J=16.4,5.2 \mathrm{~Hz}, \mathrm{H}_{\beta}\right), 2.30\left(3 \mathrm{H}, \mathrm{s}, \mathrm{CH}_{3}\right)$; $\mathrm{NMR}^{13} \mathrm{C}\left(\mathrm{CDCl}_{3}\right) \delta: 172.6(\mathrm{COOR}), 167.3(\mathrm{CON}), 142.3$ $\left(\mathrm{C}_{\mathrm{Ar}}\right), 136.3\left(\mathrm{C}_{\mathrm{Ar}}\right), 130.9\left(\mathrm{C}_{\mathrm{Ar}}\right), 129.3\left(\mathrm{C}_{\mathrm{Ar}}\right), 127.7\left(\mathrm{C}_{\mathrm{Ar}}\right), 127.2\left(\mathrm{C}_{\mathrm{Ar}}\right), 123.2\left(\mathrm{C}_{\mathrm{Ar}}\right), 122.1\left(\mathrm{C}_{\mathrm{Ar}}\right), 119.6\left(\mathrm{C}_{\mathrm{Ar}}\right), 118.5$ $\left(\mathrm{C}_{\mathrm{Ar}}\right), 111.6\left(\mathrm{C}_{\mathrm{Ar}}\right), 109.6\left(\mathrm{C}_{\mathrm{Ar}}\right), 53.7\left(\mathrm{C}_{\alpha}\right), 52.5\left(\mathrm{CH}_{3} \mathrm{O}\right), 27.7\left(\mathrm{C}_{\beta}\right), 21.5\left(\mathrm{CH}_{3}-\mathrm{Ar}\right)$. HRMS-FAB calcd. for $\mathrm{C}_{20} \mathrm{H}_{21} \mathrm{~N}_{2} \mathrm{O}_{3}[\mathrm{M}+\mathrm{H}]^{+}: 337.1552$, found: 337.1535 .

$\mathrm{N}$-(2,4,6-trimethylbenzoyl)-L-tryptophan methyl ester (14). Purification by column chromatography (hexane-EtOAc, 80:20) gave 14 as a yellow oil (109 mg, $30 \%$ yield); IR $\left(\mathrm{cm}^{-1}\right): 3279(\mathrm{NH}), 1738\left(\mathrm{CO}_{2} \mathrm{R}\right)$, $1640(\mathrm{CONH})$; $\mathrm{NMR}{ }^{1} \mathrm{H}\left(\mathrm{CDCl}_{3}\right) \delta: 8.57\left(1 \mathrm{H}, \mathrm{brs}, \mathrm{NH}_{\text {indol }}\right), 7.53\left(1 \mathrm{H}, \mathrm{d}, J=8.0 \mathrm{~Hz}, \mathrm{H}_{\mathrm{Ar}}\right), 7.24(1 \mathrm{H}, \mathrm{d}, J=8.0$ $\left.\mathrm{Hz}, \mathrm{H}_{\mathrm{Ar}}\right), 7.16-7.03\left(2 \mathrm{H}, \mathrm{m}, \mathrm{H}_{\mathrm{Ar}}\right), 6.91\left(1 \mathrm{H}, \mathrm{d}, J=2.4 \mathrm{~Hz}, \mathrm{H}_{\mathrm{Ar}}\right), 6.75\left(2 \mathrm{H}, \mathrm{s}, \mathrm{H}_{\mathrm{Ar}}\right), 6.13(1 \mathrm{H}, \mathrm{d}, J=8.0 \mathrm{~Hz}, \mathrm{NH})$, $5.18\left(1 \mathrm{H}\right.$, ddd $\left.J=8.0,6.8,5.7 \mathrm{~Hz}, \mathrm{H}_{\alpha}\right), 3.69\left(3 \mathrm{H}, \mathrm{s}, \mathrm{CH}_{3} \mathrm{O}\right), 3.36\left(1 \mathrm{H}, \mathrm{dd}, J=14.8,5.7 \mathrm{~Hz}, \mathrm{H}_{\beta}\right), 3.31(1 \mathrm{H}, \mathrm{dd}, J=$ 14.8, $6.8 \mathrm{~Hz}, \mathrm{H}_{\beta}$ ) $, 2.22\left(3 \mathrm{H}, \mathrm{s}, p-\mathrm{CH}_{3} \mathrm{Ar}\right), 2.11\left(6 \mathrm{H}, \mathrm{s}, o-\mathrm{CH}_{3} \mathrm{Ar}\right)$; $\mathrm{NMR}{ }^{13} \mathrm{C}\left(\mathrm{CDCl}_{3}\right) \delta: 172.6(\mathrm{COOR}), 170.6$ $(\mathrm{CON}), 138.7\left(\mathrm{C}_{\mathrm{Ar}}\right), 136.4\left(\mathrm{C}_{\mathrm{Ar}}\right), 134.5\left(\mathrm{C}_{\mathrm{Ar}}\right), 134.2\left(\mathrm{C}_{\mathrm{Ar}}\right), 128.3\left(\mathrm{C}_{\mathrm{Ar}}\right), 127.4\left(\mathrm{C}_{\mathrm{Ar}}\right), 123.0\left(\mathrm{C}_{\mathrm{Ar}}\right), 122.2\left(\mathrm{C}_{\mathrm{Ar}}\right)$, $119.6\left(\mathrm{C}_{\mathrm{Ar}}\right), 118.5\left(\mathrm{C}_{\mathrm{Ar}}\right), 111.5\left(\mathrm{C}_{\mathrm{Ar}}\right), 109.7\left(\mathrm{C}_{\mathrm{Ar}}\right), 52.6\left(\mathrm{C}_{\alpha} \mathrm{CH}_{3} \mathrm{O}\right), 28.0\left(\mathrm{C}_{\beta}\left(p-\mathrm{CH}_{3}-\mathrm{Ar}\right), 19.0\left(o-\mathrm{CH}_{3}-\mathrm{Ar}\right)\right.$. HRMS-FAB calcd. for $\mathrm{C}_{22} \mathrm{H}_{25} \mathrm{~N}_{2} \mathrm{O}_{3}[\mathrm{M}+\mathrm{H}]^{+}: 365.1865$, found: 365.1839 .

$\mathrm{N}$-(4-acetylbenzoyl)-L-tryptophan methyl ester (15). Purification by column chromatography (hexaneEtOAc, 60:40) gave 15 as a yellow solid $\left(1.2 \mathrm{~g}, 48 \%\right.$ yield); mp 126-129 ${ }^{\circ} \mathrm{C}$; IR $\left(\mathrm{cm}^{-1}\right): 3355(\mathrm{NH}), 1727$ $\left(\mathrm{CO}_{2} \mathrm{R}\right), 1682(\mathrm{CONH}) ; \mathrm{NMR}^{1} \mathrm{H}\left(\mathrm{CDCl}_{3}\right) \delta: 8.28\left(1 \mathrm{H}, \mathrm{brs}, \mathrm{NH}_{\text {indol }}\right), 7.93\left(2 \mathrm{H}, \mathrm{d}, J=8.6 \mathrm{~Hz}, \mathrm{H}_{\mathrm{Ar}}\right), 7.74(2 \mathrm{H}, \mathrm{d}$, $\left.J=8.6 \mathrm{~Hz}, \mathrm{H}_{\mathrm{Ar}}\right), 7.54\left(1 \mathrm{H}, \mathrm{dd}, J=8.0,0.8 \mathrm{~Hz}, \mathrm{H}_{\mathrm{Ar}}\right), 7.36\left(1 \mathrm{H}, \mathrm{dt}, J=8.2,0.8 \mathrm{~Hz}, \mathrm{H}_{\mathrm{Ar}}\right), 7.19(1 \mathrm{H}, \mathrm{ddd}, J=8.0$, $\left.7.2,0.8 \mathrm{~Hz}, \mathrm{H}_{\mathrm{Ar}}\right), 7.08\left(1 \mathrm{H}, \mathrm{ddd}, J=8.0,7.2,0.8 \mathrm{~Hz}, \mathrm{H}_{\mathrm{Ar}}\right), 7.00\left(1 \mathrm{H}, \mathrm{d}, 2.4 \mathrm{~Hz}, \mathrm{H}_{\mathrm{Ar}}\right), 6.74(1 \mathrm{H}, \mathrm{d}, J=7.6 \mathrm{~Hz}$, $\mathrm{NH}), 5.15\left(1 \mathrm{H}, \mathrm{dt}, J=7.6,5.2 \mathrm{~Hz}, \mathrm{H}_{\alpha}\right), 3.75\left(3 \mathrm{H}, \mathrm{s}, \mathrm{CH}_{3} \mathrm{O}\right), 3.49\left(1 \mathrm{H}, \mathrm{dd}, J=15.2,5.2 \mathrm{~Hz}, \mathrm{H}_{\beta}\right), 3.44(1 \mathrm{H}, \mathrm{dd}, J=$ 15.2, 5.2 Hz, $\mathrm{H}_{\beta}$ ), $2.61\left(3 \mathrm{H}, \mathrm{s}, \mathrm{CH}_{3} \mathrm{CO}\right)$; $\mathrm{NMR}^{13} \mathrm{C}\left(\mathrm{CDCl}_{3}\right) \delta$ : 197.7 (CO), 172.4 (COOR), 166.1 (CON), 139.4 $\left(\mathrm{C}_{\mathrm{Ar}}\right), 137.8\left(\mathrm{C}_{\mathrm{Ar}}\right), 136.3\left(\mathrm{C}_{\mathrm{Ar}}\right), 128.6\left(\mathrm{C}_{\mathrm{Ar}}\right), 127.8\left(\mathrm{C}_{\mathrm{Ar}}\right), 127.6\left(\mathrm{C}_{\mathrm{Ar}}\right), 123.0\left(\mathrm{C}_{\mathrm{Ar}}\right), 122.6\left(\mathrm{C}_{\mathrm{Ar}}\right), 120.0\left(\mathrm{C}_{\mathrm{Ar}}\right), 118.7$ $\left(\mathrm{C}_{\mathrm{Ar}}\right), 111.6\left(\mathrm{C}_{\mathrm{Ar}}\right), 110.0\left(\mathrm{C}_{\mathrm{Ar}}\right), 53.8\left(\mathrm{C}_{\alpha}\right), 52.7\left(\mathrm{CH}_{3} \mathrm{O}\right), 27.7\left(\mathrm{C}_{\beta}\right), 27.0\left(\mathrm{CH}_{3} \mathrm{CO}\right)$. HRMS-FAB calcd. for $\mathrm{C}_{21} \mathrm{H}_{21} \mathrm{~N}_{2} \mathrm{O}_{4}[\mathrm{M}+\mathrm{H}]^{+}:$365.1501, found: 365.1491 .

$\mathrm{N}$-(3,4-dimethoxybenzoyl)-L-tryptophan methyl ester (16). Purification by column chromatography (hexane-EtOAc, 60:40) gave 16 as a white solid $\left(1.2 \mathrm{~g}, 84 \%\right.$ yield); mp $118-123{ }^{\circ} \mathrm{C}$; IR $\left(\mathrm{cm}^{-1}\right): 3387(\mathrm{NH})$, $1741\left(\mathrm{CO}_{2} \mathrm{R}\right), 1627(\mathrm{CONH})$; $\mathrm{NMR}{ }^{1} \mathrm{H}\left(\mathrm{CDCl}_{3}\right) \delta: 8.45\left(1 \mathrm{H}\right.$, brs, $\left.\mathrm{NH}_{\text {indol }}\right), 7.55\left(1 \mathrm{H}, \mathrm{d}, J=8.0 \mathrm{~Hz}, \mathrm{H}_{\mathrm{Ar}}\right), 7.33$ $\left(1 \mathrm{H}, \mathrm{dd}, J=8.4,0.8 \mathrm{~Hz}, \mathrm{H}_{\mathrm{Ar}}\right), 7.27\left(1 \mathrm{H}, d, J=2.0 \mathrm{~Hz}, \mathrm{H}_{\mathrm{Ar}}\right), 7.19-7.15\left(2 \mathrm{H}, \mathrm{m}, \mathrm{H}_{\mathrm{Ar}}\right), 7.08(1 \mathrm{H}, \mathrm{ddd}, J=7.6,6.8$, $\left.1.2 \mathrm{~Hz}, \mathrm{H}_{\mathrm{Ar}}\right), 6.97\left(1 \mathrm{H}, \mathrm{d}, J=2.4 \mathrm{~Hz}, \mathrm{H}_{\mathrm{Ar}}\right), 6.75\left(1 \mathrm{H}, \mathrm{d}, J=8.4 \mathrm{~Hz}, \mathrm{H}_{\mathrm{Ar}}\right), 6.63(1 \mathrm{H}, \mathrm{d}, J=8.0 \mathrm{~Hz}, \mathrm{NH}), 5.13(1 \mathrm{H}$, $\left.\mathrm{dt}, J=8.0,5.2 \mathrm{~Hz}, \mathrm{H}_{\alpha}\right), 3.87\left(3 \mathrm{H}, \mathrm{s}, \mathrm{CH}_{3} \mathrm{OAr}\right), 3.80\left(3 \mathrm{H}, \mathrm{s}, \mathrm{CH}_{3} \mathrm{OAr}\right), 3.72\left(3 \mathrm{H}, \mathrm{s}, \mathrm{CH}_{3} \mathrm{O}\right), 3.46(1 \mathrm{H}, \mathrm{dd}, J=14.8$, $\left.5.2 \mathrm{~Hz}, \mathrm{H}_{\beta}\right), 3.41\left(1 \mathrm{H}, \mathrm{dd}, J=14.8,5.2 \mathrm{~Hz}, \mathrm{H}_{\beta}\right)$; $\mathrm{NMR}^{13} \mathrm{C}\left(\mathrm{CDCl}_{3}\right) \delta: 172.7$ (COOR), $167.7(\mathrm{CON}), 152.0\left(\mathrm{C}_{\mathrm{Ar}}\right)$, $149.0\left(\mathrm{C}_{\mathrm{Ar}}\right), 136.3\left(\mathrm{C}_{\mathrm{Ar}}\right), 127.8\left(\mathrm{C}_{\mathrm{Ar}}\right), 126.4\left(\mathrm{C}_{\mathrm{Ar}}\right), 123.1\left(\mathrm{C}_{\mathrm{Ar}}\right), 122.3\left(\mathrm{C}_{\mathrm{Ar}}\right), 120.0\left(\mathrm{C}_{\mathrm{Ar}}\right), 119.8\left(\mathrm{C}_{\mathrm{Ar}}\right), 118.7\left(\mathrm{C}_{\mathrm{Ar}}\right)$, 111.5 $\left(\mathrm{C}_{\mathrm{Ar}}\right), 110.5\left(\mathrm{C}_{\mathrm{Ar}}\right), 110.3\left(\mathrm{C}_{\mathrm{Ar}}\right), 110.0\left(\mathrm{C}_{\mathrm{Ar}}\right), 56.1\left(\mathrm{CH}_{3} \mathrm{OAr}\right), 56.0\left(\mathrm{CH}_{3} \mathrm{OAr}\right), 53.7\left(\mathrm{C}_{\alpha}\right), 52.6\left(\mathrm{CH}_{3} \mathrm{O}\right)$, $27.7\left(\mathrm{C}_{\beta}\right)$. HRMS-FAB calcd. for $\mathrm{C}_{21} \mathrm{H}_{23} \mathrm{~N}_{2} \mathrm{O}_{5}[\mathrm{M}+\mathrm{H}]^{+}: 383.1607$, found: 383.1643 .

$\mathrm{N}$-(4-hydroxy-3,5-dimethoxybenzoyl)-L-tryptophan methyl ester (17). Purification by column chromatography (EtOAc-hexane, 60:40) gave 17 as a yellow solid $\left(203 \mathrm{mg}, 51 \%\right.$ yield); mp $98-102{ }^{\circ} \mathrm{C}$; IR $\left(\mathrm{cm}^{-1}\right): 3377(\mathrm{NH}), 1731\left(\mathrm{CO}_{2} \mathrm{R}\right), 1642(\mathrm{CONH})$; $\mathrm{NMR}{ }^{1} \mathrm{H}\left(\mathrm{CDCl}_{3}\right) \delta: 8.83\left(1 \mathrm{H}\right.$, brs, $\left.\mathrm{NH}_{\text {indol }}\right), 7.55(1 \mathrm{H}, \mathrm{d}, J=$ $\left.8.0 \mathrm{~Hz}, \mathrm{H}_{\mathrm{Ar}}\right), 7.30\left(1 \mathrm{H}, \mathrm{d}, J=8.4 \mathrm{~Hz}, \mathrm{H}_{\mathrm{Ar}}\right), 7.17-6.99\left(2 \mathrm{H}, \mathrm{m}, \mathrm{H}_{\mathrm{Ar}}\right), 6.95\left(1 \mathrm{H}, \mathrm{d}, J=1.6 \mathrm{~Hz}, \mathrm{H}_{\mathrm{Ar}}\right), 6.83(2 \mathrm{H}, \mathrm{s}$, $\left.\mathrm{H}_{\mathrm{Ar}}\right), 6.67(1 \mathrm{H}, \mathrm{d}, J=8.0 \mathrm{~Hz}, \mathrm{NH}), 6.11(1 \mathrm{H}, \mathrm{sa}, \mathrm{OH}), 5.10\left(1 \mathrm{H}, \mathrm{dt}, J=8.0,5.2 \mathrm{~Hz}, \mathrm{H}_{\alpha}\right), 3.71\left(3 \mathrm{H}, \mathrm{s}, \mathrm{CH}_{3} \mathrm{OAr}\right)$, $3.67\left(6 \mathrm{H}, \mathrm{s}, \mathrm{CH}_{3} \mathrm{OAr}, \mathrm{CH}_{3} \mathrm{O}\right), 3.43\left(1 \mathrm{H}, \mathrm{dd}, J=14.8,5.2 \mathrm{~Hz}, \mathrm{H}_{\beta}\right), 3.38\left(1 \mathrm{H}, \mathrm{dd}, J=14.8,5.2 \mathrm{~Hz}, \mathrm{H}_{\beta}\right)$; $\mathrm{NMR}^{13} \mathrm{C}$ 
$\left(\mathrm{CDCl}_{3}\right) \delta: 172.8(\mathrm{COOR}), 167.1(\mathrm{CON}), 146.8\left(\mathrm{C}_{\mathrm{Ar}}\right), 136.2\left(\mathrm{C}_{\mathrm{Ar}}\right), 136.3\left(\mathrm{C}_{\mathrm{Ar}}\right), 127.8\left(\mathrm{C}_{\mathrm{Ar}}\right), 124.7\left(\mathrm{C}_{\mathrm{Ar}}\right), 123.2$ $\left(\mathrm{C}_{\mathrm{Ar}}\right), 122.1\left(\mathrm{C}_{\mathrm{Ar}}\right), 119.7\left(\mathrm{C}_{\mathrm{Ar}}\right), 118.4\left(\mathrm{C}_{\mathrm{Ar}}\right), 111.6\left(\mathrm{C}_{\mathrm{Ar}}\right), 109.6\left(\mathrm{C}_{\mathrm{Ar}}\right), 104.4\left(\mathrm{C}_{\mathrm{Ar}}\right), 56.3\left(\mathrm{CH}_{3} \mathrm{OAr}\right), 53.9\left(\mathrm{C}_{\alpha}\right)$, $52.5\left(\mathrm{CH}_{3} \mathrm{O}\right), 27.4\left(\mathrm{C}_{\beta}\right)$. HRMS-FAB calcd. for $\mathrm{C}_{21} \mathrm{H}_{23} \mathrm{~N}_{2} \mathrm{O}_{6}[\mathrm{M}+\mathrm{H}]^{+}:$399.1556, found: 399.1577.

$\mathrm{N}$-Benzoyl-L-isoleucine methyl ester (19). Purification by column chromatography (hexane-EtOAc, $80: 20)$ gave 19 as a white solid ( $72 \mathrm{mg}, 29 \%$ yield); mp $90.4-91.5{ }^{\circ} \mathrm{C}$; Characterization of 19 has been previously reported in the literature [25d].

\section{General Procedure for the synthesis of $N$-benzoylamino acids $(8,18,20,23)$}

A solution of the corresponding $\alpha$-aminoester $(1 \mathrm{mmol})$, benzoic anhydride $(1 \mathrm{mmol})$ and $\mathrm{AcOH}(25$ $\mathrm{mL}$ ) was refluxed for $2 \mathrm{~h}$. After reaching room temperature, the solvent was evaporated under reduced pressure and the residue was purified as indicated in each case.

$\boldsymbol{N}$-Benzoyl-L-valine (8). Purification by column chromatography (hexane-EtOAc, 70:30) gave $\mathbf{8}$ as a solid (155 mg, $70 \%$ yield); mp $127.0-127.7^{\circ} \mathrm{C}$; Characterization of 8 has been previously reported in the literature [25e].

N-Benzoyl-L-tryptophan (18). Purification by column chromatography (hexane-AcOEt, 70:30) gave 18 as oil (231 mg, $75 \%$ yield); Characterization of $\mathbf{1 8}$ has been previously reported in the literature [25e].

$\mathrm{N}$-Benzoyl-L-isoleucine (20). Purification by column chromatography (hexane-AcOEt, 50:50) gave 20 as a white solid (73 mg, $31 \%$ yield); $\mathrm{mp} 119.0-120.0{ }^{\circ} \mathrm{C}$; Characterization of 20 has been previously reported in the literature [25e].

N-Benzoyl-L-phenylalanine (23). Purification by column chromatography (hexane-AcOEt, 70:30) gave 23 as a white solid (126 mg, $47 \%$ yield); mp $141-141.5^{\circ} \mathrm{C}$; Characterization of $\mathbf{2 3}$ has been previously reported in the literature [25e].

Full experimental details, characterization data and isolated yields for all compounds were described in the "Supplementary Content" section.

\section{Antifungal Activity Evaluation}

The antifungal activity was evaluated according to the Clinical Laboratory Standard Institute (CLSI), M38A document [26]. Title compounds $(100 \mu \mathrm{L}, 640 \mu \mathrm{g} / \mathrm{mL})$ were incubated with $100 \mu \mathrm{L}$ of the fungal inoculum $\left(48 \mathrm{~h}, 35^{\circ} \mathrm{C}\right)$. After incubation, optical density was measured at $570 \mathrm{~nm}$. Results are expressed as the mean \pm SD. The inhibition percentage was calculated according to the following formula:

$$
\% \text { inhibition }=100-\left[\frac{\left(O D_{\text {treatment }}-O D_{\text {blank }}\right)(100)}{O D_{\text {control }}-O D_{\text {blank }}}\right]
$$

\section{Calculations of Physicochemical Properties}

cLogP values and Lipinsky rules criteria were calculated using SwissADME provided by the Swiss Institute of Bioinformatics [27,28].

\section{Molecular Docking Studies}

A docking study was carried out for all the $N$-benzoyl amino acid derivatives using AutodockVina software [29]. X-ray crystal structure of chitinase protein with the ligand 2-acetamido-2-deoxy- $\beta$-Dglucopyranose was obtained from the Protein Data Bank (PDB ID: 5WV9). Comparison of the poses obtained by the AutoDock Vina program with those of the crystallized protein yielded root mean square deviation $(\mathrm{RMSD})=2.094 \AA$, indicating an appropriate optimization score. 
The docking of with each ligand ( $N$-benzoyl amino derivatives) was simulated using the program AutoDock Vina, which has been used to estimate the conformation of protein-ligand complexes.

All calculations for protein-fixed ligand-flexible docking were analyzed using the Lamarckian Genetic Algorithm (LGA) method. The docking site on chitinase was defined by establishing a grid box using Pymol 3.1. The grid box size for the coordinates $x, y$, and $z$ was $60 \AA$, with a grid spacing of $0.375 \AA$, centered on $x=$ $-19.693, y=18.902$, and $z=25.671 \AA$. The best conformation was chosen based on the lowest binding energy after the docking search was completed. In the AutoDock Vina configuration files, the parameter number modes were set to 1000 modes and exhaustiveness to 1000. And for each run, the best pose was saved. The average binding energy for the best poses was used as the final binding energy value. This process was repeated ten times.

\section{Results and discussion}

\section{Chemistry}

The synthetic route for accessing $N$-benzoylamino methyl esters 1-7, 9-17 and 19 (Table 1) is illustrated in Scheme 1(a). First, 2-amino methyl esters intermediates were obtained from the reaction of a commercially-available amino acid (Val, Ile, Leu, Ala, Phe, Trp), TMSCl and methanol at room temperature [30]. Then, benzoylamino methyl esters were prepared from the corresponding benzoic acid derivative and 2amino methyl esters, employing EDAC as a peptide coupling reagent, in the presence of triethylamine and DMAP in dichloromethane [30]. $N$-benzoyl amino acids $(\mathbf{8}, \mathbf{1 8}, \mathbf{2 0 - 2 3})$ were synthesized (Scheme 1(b)) using benzoic anhydride, acetic acid and the corresponding amino acid following an optimized method described in the literature [32].

a)<smiles>[R]C(=O)NC([R2])C(=O)OC</smiles>

b)<smiles>[R2]C(N)C(=O)O</smiles>

(i) EDAC, TEA, DMAP, $\mathrm{CH}_{2} \mathrm{Cl}_{2}$; (ii) $\mathrm{Bz}_{2} \mathrm{O}, \mathrm{AcOH}$

Scheme 1. Synthetic procedure to obtain $N$-benzoylamino acid methyl esters and $N$-benzoylamino acids.

${ }^{1} \mathrm{H}$ and ${ }^{13} \mathrm{C}$ NMR spectra of compounds $\mathbf{1}, \mathbf{5}, \mathbf{8}, \mathbf{9}, \mathbf{1 8}-\mathbf{2 3}$ are in agreement with the proposed structures of the amino acid derivatives and they are in accordance with those reported in the literature [25]. Characterization of compounds $\mathbf{2 - 4 , 6 , 7 , 1 0 - 1 7}$ have not been previously described. NMR signals assigned to the $\mathrm{H} \alpha$ protons of the hitherto unknown valine derivatives were located at 4.74, 4.77 and $4.55 \mathrm{ppm}$. Methyl ester protons appeared in the range of 3.67-3.78 ppm. ${ }^{13} \mathrm{C}$ spectral data of 2,3 and 4 showed the presence of a characteristic ester group at $172.5,173.0$, and $172.8 \mathrm{ppm}$, while the amide functionality was confirmed by the signals at 167.2, 167.0, and $172.5 \mathrm{ppm}$, respectively. Regarding tryptophan derivatives $(\mathbf{6 , 7 , 1 0 - 1 7 ) , ~ H \alpha ~ s i g n a l s ~}$ were found in the range of $4.78-5.18 \mathrm{ppm}$, while $\mathrm{C} \alpha$ resonances were observed at 52.6-57.5 ppm. 
Table 1. Synthesized Compounds.

\begin{tabular}{|c|c|c|c|c|}
\hline & O & $\operatorname{l}_{\mathrm{CO}_{2} \mathrm{R}^{3}}^{\mathrm{R}^{2}}$ & & \\
\hline \multirow{2}{*}{ Entry } & \multirow{2}{*}{$\mathbf{R}^{1}$} & $\mathbf{R}^{2}$ & \multirow{2}{*}{$\mathbf{R}^{3}$} & \multirow{2}{*}{$\begin{array}{c}\text { Yield } \\
(\%)\end{array}$} \\
\hline & & Valine derivatives & & \\
\hline 1 & Phenyl & \multirow{4}{*}{ isopropyl } & $\mathrm{Me}$ & 85 \\
\hline 2 & 3-Methoxyphenyl & & $\mathrm{Me}$ & 75 \\
\hline 3 & 3,4-Dimethoxyphenyl & & $\mathrm{Me}$ & 75 \\
\hline 4 & 2-(4-hydroxyphenyl)acetyl & & $\mathrm{Me}$ & 86 \\
\hline 5 & Phenyl & $(R)$-isopropyl & $\mathrm{Me}$ & 85 \\
\hline 6 & 4-Methylphenyl & \multirow{3}{*}{ isopropyl } & $\mathrm{Me}$ & 99 \\
\hline 7 & 2,4,6-Trimethylphenyl & & $\mathrm{Me}$ & 36 \\
\hline 8 & Phenyl & & $\mathrm{H}$ & 70 \\
\hline & & Tryptophan derivatives & & \\
\hline 9 & Phenyl & \multirow{10}{*}{$\mathrm{CH}_{2}$-(3-indolyl) } & $\mathrm{Me}$ & 45 \\
\hline 10 & 2-Hydroxyphenyl & & $\mathrm{Me}$ & 58 \\
\hline 11 & 2-Hydroxy-3-pyridyl & & $\mathrm{Me}$ & 43 \\
\hline 12 & 2-Chloro-3-pyridyl & & $\mathrm{Me}$ & 28 \\
\hline 13 & 4-Methylphenyl & & $\mathrm{Me}$ & 76 \\
\hline 14 & 2,4,6-Trimethylphenyl & & $\mathrm{Me}$ & 30 \\
\hline 15 & 4-Acetylphenyl & & $\mathrm{Me}$ & 48 \\
\hline 16 & 3,4-Dimethoxyphenyl & & $\mathrm{Me}$ & 84 \\
\hline 17 & $\begin{array}{c}\text { 4-Hydroxy-3,5- } \\
\text { dimethoxyphenyl }\end{array}$ & & $\mathrm{Me}$ & 51 \\
\hline \multirow[t]{2}{*}{18} & Phenyl & & $\mathrm{H}$ & 75 \\
\hline & & \multirow{3}{*}{$\begin{array}{l}\text { Isoleucine derivatives } \\
(1 S)-1-\text { methylpropyl }\end{array}$} & & \\
\hline 19 & Phenyl & & $\mathrm{Me}$ & 29 \\
\hline \multirow[t]{2}{*}{20} & Phenyl & & $\mathrm{H}$ & 31 \\
\hline & & Leucine derivative & & \\
\hline \multirow[t]{2}{*}{21} & Phenyl & isobutyl & $\mathrm{H}$ & 29 \\
\hline & & Alanine derivative & & \\
\hline \multirow[t]{2}{*}{22} & Phenyl & methyl & $\mathrm{H}$ & 71 \\
\hline & & Phenylalanine derivative & & \\
\hline 23 & Phenyl & benzyl & $\mathrm{H}$ & 47 \\
\hline
\end{tabular}

\section{Structure-activity relationships}

All compounds prepared herein were tested for their in vitro growth inhibition activity against two filamentous fungi, F. temperatum and A. fumigatus. They are common pathogens that produce mycotoxins causing plant diseases in crops, such as sugar cane, sorghum, beans and corn. Plant infection affects all growth stages, producing lower yields [33]. Aspergillus fumigatus is an opportunist pathogen located in the environment that causes a wide range of infections including the invasive aspergillosis, the most common mold infection worldwide [34].

Analysis of data depicted in Table 2 allowed us to establish some structure-activity relationships. $N$ benzoylamino methyl esters and acids derived from valine $(\mathbf{1}, \mathbf{2}, \mathbf{5}-7)$, tryptophan $(\mathbf{1 0}-\mathbf{1 3})$, and isoleucine (19) showed good inhibition activity. The highest inhibition growth was found in compounds 7 (78.2 \%) and $\mathbf{1 3}$ (78.5 \%) against $A$. fumigatus and $F$. temperatum, respectively. Remarkably, $N$-benzoylamino methyl esters (1, 
$2,5,6,7,10$, and 13) were potent antifungal agents whereas, $N$-benzoyl amino acids were inactive at the tested concentration. Furthermore, the activity also depends on the side chain of the amino acid, being valine $(\mathbf{1}, \mathbf{2}, \mathbf{5}$, 6, and 7) and two tryptophan (10 and 13) derivatives the most active against A. fumigatus. Moreover, 10 and 13 were also the most potent ones against $F$. temperatum. Nevertheless, esters and/or acids derived from alanine, leucine, isoleucine, and phenylalanine exhibited moderate or no activity $(<50 \%)$, except compound 19, which showed good inhibitory activity against $F$. temperatum $(52.6 \%)$.

Among $N$-benzoyl esters derivatives from valine, compound 7, which bears 3 methyl substituents on the aromatic ring, exhibited the highest inhibitory activity against both fungi. Comparison of compounds $\mathbf{1}, \mathbf{6}$, and 7, revealed that an increase in the number of methyl groups located on the aromatic ring affords an improvement of the activity, particularly in F. temperatum. This effect might probably be due to an increment in the lipophilicity (cLogP: 2.48, 3.05). This feature is an important physicochemical property for the bioavailability of the compound. Moreover, change in the stereochemistry of $\mathrm{C} \alpha(\mathbf{1} v s$. 5) enhanced the inhibitory properties. On the contrary, compound 3, bearing 2 methoxy groups exhibited the lowest activity against $A$. fumigatus within valine ester derivatives, followed by $\mathbf{4}$, that contains a hydroxyl moiety. Thus, isosteric replacement (Me vs. $\mathrm{OH})$, enhanced the antifungal activity.

Concerning $N$-benzoyl tryptophan derivatives, the presence of a heterocyclic moiety (pyridine) diminished the activity against both fungi (10 vs. 11). Incorporation of a single electron donating substituent (10 and 13) afforded the most potent compounds of this series. No violations of Lipinsky's rule were found for compounds included in Table 2 .

Table 2. Biological activity and selected computational results.

\begin{tabular}{|c|c|c|c|c|c|}
\hline \multicolumn{3}{|c|}{ Inhibition Activity $(\%)^{\mathrm{a}}$} & \multicolumn{3}{|c|}{ Computational results } \\
\hline Valine derivatives & A. fumigatus & $\begin{array}{c}F . \\
\text { temperatum }\end{array}$ & cLog P & $\begin{array}{c}\text { Docking } \\
\text { Score } \\
(\mathrm{kcal} / \mathrm{mol})^{\mathrm{b}}\end{array}$ & $\begin{array}{l}\text { Predicted } \\
K_{i}(\mu \mathbf{M})^{\mathbf{b}, \mathrm{c}}\end{array}$ \\
\hline 1 & 61.5 & 25.1 & 2.15 & -7.90 & 1.54 \\
\hline 2 & 53.6 & 21.6 & 2.19 & -7.80 & 1.83 \\
\hline 3 & 23.4 & 28.3 & 2.07 & -7.73 & 2.06 \\
\hline 4 & 9.8 & 18.6 & 1.83 & -7.9 & 1.62 \\
\hline 5 & 70.7 & 68.9 & 2.16 & -7.87 & 1.62 \\
\hline 6 & 54.9 & 68.7 & 2.48 & -8.51 & 0.55 \\
\hline 7 & 78.2 & 71.1 & 3.05 & -8.51 & 0.55 \\
\hline 8 & 0 & 0 & 1.73 & -8.24 & 0.87 \\
\hline \multicolumn{6}{|c|}{ Tryptophan derivative } \\
\hline & 0 & 13.4 & 2.83 & -10.20 & 0.03 \\
\hline 10 & 58.0 & 74.0 & 2.09 & -9.90 & 0.05 \\
\hline 11 & 36.6 & 7.5 & 2.00 & -9.80 & 0.05 \\
\hline 12 & 6.6 & 9.0 & 2.67 & -10.1 & 0.03 \\
\hline 13 & 51.5 & 78.5 & 2.84 & -10.50 & 0.02 \\
\hline 14 & 5.2 & 0 & 3.72 & -10.40 & 0.02 \\
\hline 15 & 0.0 & 0 & 2.54 & -9.81 & 0.06 \\
\hline 16 & 28.4 & 0 & 2.83 & -9.79 & 0.06 \\
\hline 17 & 20.0 & 48.6 & 2.48 & -9.69 & 0.07 \\
\hline 18 & 0 & 0 & 2.37 & -10.40 & 0.02 \\
\hline \multicolumn{6}{|c|}{ Isoleucine derivatives } \\
\hline 19 & 24.7 & 52.6 & 2.48 & -10.40 & 0.02 \\
\hline 20 & 0 & 0 & 1.94 & -8.03 & 1.24 \\
\hline \multicolumn{6}{|c|}{ Leucine derivative } \\
\hline 21 & 0 & 0 & 2.02 & -8.03 & 1.24 \\
\hline
\end{tabular}




\begin{tabular}{|c|c|c|c|c|c|}
\hline \multicolumn{6}{|c|}{ Alanine derivative } \\
\hline 22 & 0 & 0 & 1.05 & -7.32 & 4.12 \\
\hline \multicolumn{6}{|c|}{ Phenylalanine derivative } \\
\hline 23 & 0 & 0 & 2.36 & -9.40 & 0.12 \\
\hline Amphotericin B & 73.5 & 67.0 & & & \\
\hline
\end{tabular}

${ }^{\mathrm{a}}$ Calculated at $640 \mu \mathrm{g} / \mathrm{mL}$.

${ }^{\mathrm{b} B i n d i n g}$ affinity for the interaction of glucose and synthetized derivatives with chitinase.

${ }^{\mathrm{c}} K_{\mathrm{i}}=\mathrm{e}-\Delta \mathrm{G} / \mathrm{RT} \Delta \mathrm{G}=$ Gibbs free energy; $\mathrm{R}=1.9872 \mathrm{cal} / \mathrm{mol} . \mathrm{K} ; \mathrm{T}=298.15 \mathrm{~K}$.

\section{Molecular Docking Analysis}

Taking into consideration that the antifungal activity might be due to the inhibition of fungal chitinases [35], docking calculations were performed to predict binding affinities between the enzyme and the compounds prepared herein.

Molecular docking analysis was carried out using Autodock Vina software. First, the active center validation was performed, where an RMSD of $2.094 \AA$ was obtained in one of the catalytic sites of the enzyme (Fig. S1, provided in the supplementary information).

Docking of the complex protein-ligand of reference showed thermodynamically favorable binding energy of $-6.5 \mathrm{Kcal} / \mathrm{mol}$. The stability of this complex is favored by hydrogen bonding between the hydroxyl groups of the glucopyranose and the aminoacid residues localized in the active site of the enzyme. 2-Acetamido2-deoxy- $\beta$-d-glucopyranose exhibited four hydrogen bonding interactions with four amino acids presented in the binding site of the enzyme: ARG342 (NH1), ASP286 (OD2), TYR285 (OH) and TRP176 (NB). In addition, hydrophobic interactions also contribute to stabilize energetically the ligand (Table 1, ID PDB 5WV9, provided in the supplementary information).

All the $N$-benzoyl amino acid derivatives were analyzed by molecular docking studies in order to find the interactions in the active site of the enzyme. The results revealed that title compounds show hydrogen bonding interactions with TRP176, TYR218, TYR285, TYR340, TRP433, GLU217, GLU370, ASP215, ASP286, GLY175 and ARG342 (Table 1, provided in the supplementary information); all these amino acid residues are found at the catalytic site. Similar hydrogen bonding interactions are observed between the cocrystallized ligand and the amino acid residues: ARG342 (NH1), ASP286 (OD2), TYR285 (OH) and TRP176 (NB).

Analysis of the influence of the configuration on $\mathrm{C}_{\alpha}(\mathbf{1} \mathrm{vs} . \mathbf{5})$ revealed a similar docking score energy and therefore a similar predicted $K_{i}$, in that sense, docking simulations predicted that there is no significant influence of the configuration of that center on the inhibition of chitinase. According to Table 7 of the supporting information, compound 1 established a higher number of interactions with the active site of the enzyme, however the overall energetic contribution of these interactions are similar on both compounds. This observation is in agreement with the measured biological activity against A. fumigatus $(61.5 \%$ and $70.7 \%$ growth inhibition). The fact that compound $\mathbf{1}$ is clearly less active against $F$. temperatum might suggest that chitinase is not the only therapeutic target in the activity of these compounds or that the bioavailability is not the same in both fungal strains.

According to the growth inhibition activity measured in compounds 6 and 7, it was observed that the compound with three methyl groups attached to the aromatic ring is more active for both strains than the compound with one methyl group; this might be explained due to the presence of $\pi$-alkyl lipophilic interactions in the aromatic moiety. Comparison between compounds $\mathbf{6}$ and $\mathbf{7}$ revealed that whereas for compound $\mathbf{6}$ there is a single $\pi$-alkyl interaction between the methyl of the para position and the TRP176, for derivative 7 there are three $\pi$-alkyl interactions: two with the methyl in the para position and TRP102, PHE129 and one with the methyl in the ortho position and TRP433. This might suggest that an increase of the number of methyl groups would afford a better interaction with the active site of the enzyme and therefore a better chitinase inhibition. However, overall docking energy was predicted to be the same in both cases. Although the calculations are similar for tryptophan derivatives (13 and 14) which are also predicted to have similar activity, monomethylated derivative 13 was found to be much more potent than 14. 
In valine series, the least active (3-4 and 8) compounds lack $\pi$-alkyl lipophilic interactions in the aromatic moiety.

In valine series, compounds $\mathbf{6}$ and $\mathbf{7}$ which are the most actives ones, are also predicted to be the most potent inhibitors, and therefore, to have the lowest docking energy. In this sense, the least active compounds of the series (derivatives 1-4) are also predicted to be the least efficient when interacting with the enzyme (higher docking energy).

Although we have analyzed the influence of the conformation in the amide group (Table 7, supporting information file), there is not a clear correlation between this property and the biological activity.

\section{Conclusion}

In conclusion, $N$-benzoyl amino esters constitute an attractive template for the development of new antifungal agents. To our knowledge, these compounds are the simplest amino acid derivatives with antifungal activity. Relevant antimycotic activity was found in $N$-benzoyl amino esters derived from valine or tryptophan, especially compound 7, which showed remarkable activity for the two filamentous fungi (A. fumigatus and $F$. temperatum) while five derivatives $(5-7,10,13)$ were found to be active against $F$. temperatum. Some structure-activity relationships could also be established regarding the substituent on the aromatic ring of the benzoyl moiety. In valine derivatives, the presence of a methyl group improved the activity, whereas strong electron donating or electron withdrawing groups decreased it. A molecular docking study was employed to investigate their binding towards chitinase, a potential target against fungi. We have attempted to determine the key interactions between the compounds prepared herein and the active site of chitinase, a potential target involved in the antifungal activity of such compounds. Interactions involving the aromatic rings, the influence on the number of methyl substituents, and configurations on the $\alpha$-carbon have been analyzed.

\section{Acknowledgments}

The authors thank Consejo Nacional de Ciencia y Tecnología (CONACYT) of Mexico for the award of fellowship to YCP, and financial grants (INFRA-2015-01-252013 and INFRA-2019-301144). We are grateful to Dra. María Yolanda Ríos Gómez, Ing. Victoria Labastida, and T. C. María Medina Pastor (Universidad Autónoma del Estado de Morelos, for technical assistance).

\section{References}

1. Low, C-Y.; Rotstein, C. F1000 Med. Rep. 2011, 3, 1-8 DOI: http://dx.doi.org/10.3410/M3-14

2. Davies, S. F.; Sarosi, G. A. Postgrad. Med. J. 2016, 73, 242-251. DOI: http://dx.doi.org/10.1080/00325481.1983.11697878

3. Kainz, K.; Bauer, M. A.; Madeo, F.; Carmona-Gutiérrez, D. MicroB. Cell. 2020, 7, 143-145 DOI: http://dx.doi.org/10.15698/mic2020.06.718

4. Fisher, M. C.; Nichola J. H.; N. J.; Sanglard, D.; Gurr, S. H. Science. 2018, 360, 739-742. DOI: http://dx.doi.org/10.1126/science.aap7999

5. Verweij, P. E.; Mellado, E.; Melchers W. J. G. N. Engl. J. Med. 2007, 356, 1481-1483. DOI: http://dx.doi.org/10.1056/NEJMc061720

6. Walker, L. A.; Gow, N. A. R. Munro, C. A.; Fungal Genet. Biol. 2010, 47, 117-126. DOI: http://dx.doi.org/10.1016/j.fgb.2009.09.003 
7. World Health Organization. (2020). First meeting of the WHO antifungal expert group on identifying priority fungal pathogens: meeting report. DOI: https://apps.who.int/iris/handle/10665/332309 (last accessed May, 16th, 2021).

8. Van Daele, R.; Spriet, I.; Wauters, J.; Maertens, J.; Mercier, T.; Van Hecke, S.; Brüggemann, R.; Med. Mycol. 2019, 57, 328-343. DOI: http://dx.doi.org/10.1093/mmy/myz012

9. Krebs, H. A.; Wiggins, D.; Stubbs, M.; Sols, A.; Bedoya, F. Biochem. J. 1983, 214, 657-63. DOI: http://dx.doi.org/10.1042/bj214065

10. Krátký, M.; Vinšová, J. Molecules. 2012, 17, 9426-9442. DOI: https://doi.org/10.3390/molecules17089426

11. Berne, S.; Kovaĉiĉ, L.; Sova, M.; Kraševec, N.; Gobec, S.; Križaj, I.; Komel, R. Bioorg. Med. Chem. 2015, 23, 4264-4276. DOI: http://dx.doi.org/10.1016/j.bmc.2015.06.042

12. Lima, T. C.; Ferreira, A. R.; Silva, D. F.; Lima E. O.; de Sousa D. P. Nat. Prod. Res. 2018, 32, 572575. DOI: http://dx.doi.org/doi:10.1080/14786419.2017.1317776

13. Kaminski, H. M.; Feix, J. B.; Polymers. 2011, 3, 2088-2106. DOI: http://dx.doi.org/10.3390/polym3042088

14. Shivakumara, K. N.; Prakasha, K. C.; Gowda, D. C. E-J. Chem. 2009, 6, S473-S479. DOI: http://dx.doi.org/10.1155/2009/267296

15. Garg, A.; Borah, D.; Trivedi, P.; Gogoi, D.; Chaliha, A. K.; Ali, A. A.; Chetia, D.; Chaturvedi, V. Sarma, D. ACS Omega. 2020, 5, 29830-29837. DOI: https://doi.org/10.1021/acsomega.0c03862

16. Sardina, F. J.; Rapoport, H. Chem. Rev. 1996, 96, 1825-1872. DOI: https://doi.org/10.1021/cr9300348

17. Philip, A.; Kepler, J. A.; Johnson, B. H.; Carroll. F. I. J. Med. Chem. 1988, 31, 870-874. DOI: https://doi.org/10.1021/jm00399a032

18. Oliva, F.; Bucci, R.; Tamborini, L.; Pieraccini, S.; Pinto, A.; Pellegrino, S. Front. Chem. 2019, 18, $1-$ 10. DOI: http://dx.doi.org/10.3389/fchem.2019.00133

19. Battista, N.; Bari, M.; Bisogno, T.; Biomolecules. 2019, 9, 822. DOI: http://dx.doi.org/10.3390/biom9120822

20. Duarte R. C.; Ongaratto, R.; Piovesan, L. A.; de Lima, V. R.; Soldi, V.; Merlo, A. A.; D'Oca, M. G. M. Tetrahedron Lett. 2012, 53, 2454-2460. DOI: https://doi.org/10.1016/j.tetlet.2012.03.015

21. Abdel-Rahman, A. A. H. Monatsh. Chem. 2008, 139, 289-297. DOI: http://dx.doi.org/10.1007/s00706-007-0770-7

22. Krátký, M.; Vinšová, J.; Buchta, V.; Horvati, K.; Bösze, S.; Stolaříková, J. Eur. J. Med. Chem. 2010, 45, 6106-6113. DOI: http://dx.doi.org/10.1016/j.ejmech.2010.09.040

23. Abdel-Wahab, S. M.; Abdelsamii, Z. K.; Abdel-Fattah, H. A.; El-Etrawy, A. S.; Georghiou, P. E. Int. J. Pharm. Chem. 2016, 6, 149-159. DOI: https://doi.org/10.7439/ijpc.v6i6.3292

24. Prasher, P. Chem. Sci. J. 2016, 7, 847-858. DOI: http://dx.doi.org/10.4172/2150-3494.1000139

25. a) Yoo, W-J.; Li, C-J. J. Am. Chem. Soc. 2006, 128, 13064-13065. DOI: https://doi.org/10.1021/ja064315 b) Tatar, E.; küçükgüzel, I.; Daelemans, D.; Talele, T. T.; KaushikBasu, N.; De Clercq, E. Pannecouque, C. Arch. Pharm. 2013, 346, 140-153. DOI: https://doi.org/10.1002/ardp.201200311 c) Trevitt, C. R.; Craven, C. J.; Milanesi, L.; Syson, K.; Mattinen, M-L.; Perkins, J.; Annila, A.; Hunter, C. A.; Waltho J. P. Chem. Biol. 2005, 12, 89-97. DOI: https://doi.org/10.1016/j.chembiol.2004.11.007 d) Anderson, Z, J.; Hobson, C.; Needley, R.; Song, L.; Perryman, M. S.; Kerby, P.; Fox, D. J. Org. Biomol. Chem. 2017, 15, 9372-9378. DOI: https://doi.org/10.1039/C7OB01995E e) Duddeck, H. Magn. Reson. Chem. 2009, 47, 222-227 DOI: https://doi.org/10.1002/mrc.2374 
26. National Committee for Clinical Laboratory Standards 2002. Reference Method for Broth Dilution Antifungal Susceptibility Testing of Conidium-forming Filamentous Fungi. Proposed Standard Document M38-A. Clinical and Laboratory Standards Institute. Wayne, Pa. https://clsi.org/media/1455/m38a2_sample.pdf (last accessed May, 16th, 2021).

27. SIB. SwissADME. Available online: http://www.swissadme.ch (last accessed April, 23th, 2021).

28. Daina, A.; Michielin, O.; Zoete, V. Sci. Rep. 2017, 7, 42717. DOI: https://doi.org/4271710.1038/srep42717

29. Trott, O.; Olson, A. J. J. Comput. Chem. 2010, 31, 455-461. DOI: 10.1002/jcc.21334.

30. Li, J.; Sha, Y. Molecules 2008, 13, 1111-1119. DOI: https://doi.org/10.3390/molecules13051111.

31.Pérez-Picaso, L.; Escalante, J.; Olivo, H. F.; Rios, M. Y. Molecules. 2009, 14, 2836-2849 DOI: https://doi.org/10.3390/molecules14082836

32. Martynenko, Y. V.; Kazunin, M. S.; Nosulenko, I. S.; Berest, G. G.; Kovalenko, S. I. Kamyshnyi, O. M.; Polishchuk, N. M. Zaporož. med. ž. 2018, 20, 413-420. DOI: https://doi.org/10.14739/23101210.2018.3.130544

33. Robles-Barrios K. F.; Medina-Canales M. G.; Rodríguez-Tovar A. V.; Octavio-Pérez N. Mycopathologia. 2007, 163, 31-39. DOI: https://doi.org/10.1007/s11046-006-0082-1

34. Latgé, J-P. Clin. Microbiol. Rev. 1999, 12, 310-350. DOI: https://doi.org/10.1128/CMR.00140-18

35. Li, Y.; Sun, H.; Zhu, X.; Bian, C.; Wang, Y.; Si, S. Molecules. 2019, 24, 3155; DOI:10.3390/molecules24173155 